\title{
SOBRE A EQUIVALENNCIA DE PERCENTUAIS EM INVESTIMENTOS PÓS-FIXADOS
}

\author{
ON EQUIVALENT INTEREST RATES ON POST-FIXED INVESTMENTS
}

\author{
ADRIANO RODRIGUES DE MELO ${ }^{a}$ \\ NORTON PIZZI MANASSI ${ }^{\mathrm{b}}$
}

\begin{abstract}
Resumo
Este trabalho apresenta uma aplicação da matemática financeira no desenvolvimento de três metodologias utilizadas para se determinar a equivalência entre percentuais destacados para a remuneração de títulos de renda fixa pósfixados. Tem por objetivo evidenciar as hipóteses subjacentes às construções dos três estimadores, além de estabelecer algumas desigualdades que configuram a relação matemática entre suas fórmulas. Dispõe sobre as simulações computacionais realizadas com o intuito de apurar a acurácia desses estimadores. Conclui-se que, apesar das restrições impostas aos seus usos, todos eles fornecem aproximações úteis para o propósito de conversão. Salienta-se, ademais, que tais metodologias correspondem à aproximações e que o valor verdadeiro da conversão, pautada pelo critério de equivalência, pode variar ao longo do tempo.
\end{abstract}

Palavras-chave: Taxas Equivalentes, Matemática Financeira, Investimentos.

\begin{abstract}
This paper presents the development of three methodologies used to calculate the equivalence between percentages used in post-fixed remuneration of fixed income securities. In addition to highlighting the assumptions underlying the construction of the three estimators, it establishes some inequalities that configure the mathematical relationship of formulas. This work also presents computational simulations carried out in order to verify the accuracy of these estimators. We conclude that they all provide good approximations for the
\end{abstract}

${ }^{a}$ Instituto Federal Catarinense, Araquari/Brasil; ORCID: https://orcid.org/0000-0002-30438906; E-mail: adriano.melo@ifc.edu.br

${ }^{\mathrm{b}}$ Escola de Aprendizes-Marinheiros de Santa Catarina, Florianópolis/Brasil; ORCID: https://orcid.org/0000-0002-6030-7683; E-mail: nortonmanassi@gmail.com 
purpose of equivalence. Finally, we emphasize that such methodologies correspond to approximations and that the true value of the conversion, based on the equivalence criterion, may vary over time.

Keywords: Equivalent Interest Rate, Financial Mathematics, Investments.

MSC2010: 91-01, 91G99, 97M30.

\section{Introdução}

Faz parte do escopo da matemática financeira o estudo sobre a equivalência de taxas percentuais, a qual pode ser definida nos seguintes termos [8, p. 40]:

Definicão 1. Duas taxas percentuais $i_{1}$ e $i_{2}$ são ditas equivalentes quando, ao serem aplicadas ao mesmo capital $C$, pelo mesmo tempo, geram o mesmo montante $M$.

Um uso imediato da Definição 1 ocorre quando se deseja converter taxas para períodos distintos daqueles os quais elas se referem. Por exemplo, uma taxa $i_{m}$ de período mensal pode ser convertida em outra taxa $i_{a}$ de período anual bastando, nos termos da equivalência de taxas, que se verifique a seguinte igualdade:

$$
C\left(1+i_{a}\right)=M=C\left(1+i_{m}\right)^{12}
$$

A equação (1) expressa algebricamente todo o conteúdo da Definição 1 e, portanto, sua solução $i_{a}=\left(1+i_{m}\right)^{12}-1$ representa a maneira na qual ocorre a equivalência entre taxas mensais e anuais ou, dito de outra forma, o modo de se realizar a conversão entre elas.

O tema investimentos, uma das principais áreas de aplicação da matemática financeira, constitui outra situação em que o assunto equivalência de taxas percentuais pode ser destacado. De fato, dentro da grande variedade de opções disponíveis no mercado financeiro, existem os investimentos de renda fixa pós-fixados, cuja remuneração está atrelada à variação de alguma taxa de juros do mercado [1], como a taxa SELIC ou a taxa $\mathrm{DI}^{1}$. Por exemplo, se $i_{D I}$ é a taxa DI diária, um CDB pós-fixado em $105 \%$ do DI é um título com rendimento de $1,05 i_{D I}$ ao dia. Dizemos que $\rho=105 \%$ é o percentual destacado para a remuneração.

\footnotetext{
${ }^{1}$ São exemplos o CDB (Certificado de Depósito Bancário), a LCI (Letra de Crédito Imobiliário) e a LCA (Letra de Crédito do Agronegócio), mais comumente indexados à taxa DI e também o título público LFT (Letra Financeira do Tesouro), também conhecido como Tesouro Selic, vinculado à taxa básica de juros da economia, a taxa SELIC.
} 
$\mathrm{Na}$ hipótese de estarmos interessados em decidir entre dois investimentos, atrelados a um mesmo indexador através dos percentuais destacados $\rho_{1}$ e $\rho_{2}$, tendo como critério o maior retorno, deparamo-nos com as seguintes situações: i) se os investimentos possuem mesma regra de tributação, a decisão gira em torno dos percentuais $\rho_{1}$ e $\rho_{2}$, neste caso, o maior superará em ganhos; ii) se a regra de tributação for distinta, então passa a ser interessante obter uma relação entre $\rho_{1}$ e $\rho_{2}$ que considere o imposto devido.

De maneira mais direta, a situação ii) pode ser expressa assim: dado um capital $C$ e dois produtos de renda fixa pós-fixados $Q$ e $P$, ambos indexados a uma mesma taxa de juros em $q$ e $p$, respectivamente. Suponha que sobre o primeiro incida imposto de renda (IR) e sobre o segundo não. Como decidir sobre $Q$ e $P$ ? $\mathrm{Ou}$ ainda, a partir de qual valor de $p$ o produto não isento passa a ser vantajoso frente ao isento? A análise deste problema exige o estabelecimento da equivalência entre os referidos percentuais.

Diante do contexto deste problema, justificamos o presente trabalho pelo fato de não termos encontrado na literatura acadêmica o desenvolvimento do presente tema, apesar de termos identificado suas utilizações pelos meios de comunicação e informação, no que se refere à comparações desta natureza. Por essa razão, este trabalho tem como objetivo levantar uma discussão acerca dos fundamentos de tais metodologias de cálculo, buscando evidenciar as hipóteses subjacentes às suas construções.

No decorrer da leitura, na seção 2, descrevemos o desenvolvimento das estratégias. Na seção 3, buscamos estabelecer relações entre as três formas de cálculo, enquanto que na seção 4, dedicamos à apresentação de experimentos computacionais que simulam a utilização dessas três estratégias. A seção 5 dispõe sobre as conclusões obtidas tanto pela formalização matemática quanto pelas simulações computacionais.

\section{Algumas Estimativas Para Equivalência de Per- centuais}

Para iniciar a discussão, começamos por definir a equivalência de percentuais atrelados a uma taxa $i$, o que faremos de maneira análoga à Definição 1:

Definicão 2. Dois percentuais $\rho_{1} e \rho_{2}$, destacados para a remuneração, sobre a qual incide uma taxa $i$, são ditos equivalentes quando, ao serem aplicados ao mesmo capital, pelo mesmo tempo, geram o mesmo montante líquido. 


\subsection{Estimativas Baseadas na Taxa Acumulada}

A primeira estimativa apresentada se apoia na hipótese de que os percentuais $p$ e $q$, associados aos investimentos $P$ (isento) e $Q$ (não isento), respectivamente, podem incidir diretamente sobre a taxa de juros acumulada no período. Dessa forma, seja $i_{a}$ a referida taxa acumulada e $i_{r}$ a alíquota do imposto de renda devido, então sobre a hipótese enunciada, a taxa de juros do período corrigida pelos percentuais $p$ e $q$ se torna $p i_{a}$ e $q i_{a}$ e os rendimentos líquidos sobre os produtos $P$ e $Q$ são dados, respectivamente, por $J_{P}=p i_{a} C$ e $J_{Q}=q i_{a} C\left(1-i_{r}\right)$, onde $C$ é o capital disponível para o investimento. Gostaríamos, para efeito de equivalência e nos termos da Definição 2, que $J_{P}$ e $J_{Q}$ fossem iguais, ou seja,

$$
p i_{a} C=J_{P}=J_{Q}=q i_{a} C\left(1-i_{r}\right)
$$

que nos leva à relação

$$
p=\left(1-i_{r}\right) q
$$

A fórmula dada pela equação (2) é muito presente em sites com conteúdos que tratam do tema associado à investimentos em Renda Fixa. A vantagem dessa fórmula é sua simplicidade, exemplificada a seguir.

Exemplo 1. Para um período $n$ acima de 720 dias úteis, a alíquota de IR será de $i_{r}=15 \%$ sobre o rendimento, conforme Tabela 1 , então um investimento $Q$ pós-fixado em $q=115 \%$ da taxa DI equivaleria, nos termos da fórmula (2), a um investimento $P$ de $p=97,75 \%$ do DI. Isso significa que, considerando apenas a remuneração, um investimento isento (como uma LCI) deve remunerar algo superior a 97,75\% da taxa DI para que seja vantajoso em relação a um investimento tributado (como um CDB) que paga $115 \%$ do DI.

Tabela 1: Tabela de imposto sobre rendimentos de capital.

\begin{tabular}{c|c}
\hline Prazo & Alíquota \\
\hline Até 180 dias & $22,5 \%$ \\
181 até 360 dias & $20 \%$ \\
361 até 720 dias & $17,5 \%$ \\
Acima de 720 dias & $15 \%$ \\
\hline \multicolumn{2}{c}{ Fonte: Ministério da Economia [6]. }
\end{tabular}

No Exemplo 1, utilizamos a equação (2) para obter um valor aproximado de $p$ em termos de $q$. Trata-se de uma aproximação por que a hipótese utilizada em sua 
dedução não condiz com a realidade, uma vez que o cálculo da taxa DI, acumulada entre datas e ajustada por um percentual $\rho$ destacado para remuneração, é efetuado através da seguinte fórmula [4]:

$$
i_{a}=\prod_{j=1}^{n}\left(1+\rho i_{j}\right)-1,
$$

em que $i_{j}$ corresponde à $j$-ésima taxa DI diária. Ou seja, a atualização mediante o percentual destacado ocorre diariamente e não sobre o período acumulado. Dessa forma, usaremos $\hat{p}_{1}$ para nos referirmos ao resultado obtido pela equação (2), indicando que se trata de uma estimativa para $p$. Na seção seguinte apresentaremos outro estimador, agora baseando na equação (3).

\subsection{Estimativa Baseada no Rendimento Líquido Acumulado}

Outro estimador pode ser construído ao considerar-se o rendimento líquido acumulado. Sabendo que a taxa acumulada é dada por (3), podemos obter a taxa de juros líquida do investimento $Q$, acumulada no período, por meio da razão:

$$
i_{a}=\frac{J_{Q}}{C}=\frac{\left(1-i_{r}\right) C\left[\prod_{j=1}^{n}\left(1+q i_{j}\right)-1\right]}{C}=\left(1-i_{r}\right)\left[\prod_{j=1}^{n}\left(1+q i_{j}\right)-1\right] .
$$

Ora, dividindo $i_{a}$ pelo índice DI acumulado no período $n$, obteremos uma proporção do acumulado em termos do DI, isto é, obteremos a estimativa dada por:

$$
\hat{p}_{2}=\frac{\left(1-i_{r}\right)\left[\prod_{j=1}^{n}\left(1+q i_{j}\right)-1\right]}{\prod_{j=1}^{n}\left(1+i_{j}\right)-1} .
$$

Para exemplificar a fórmula em (4), simularemos um produto de renda fixa indexado ao DI.

Exemplo 2. Consideremos um CDB de $q=115 \%$ do DI aplicado pelo período de 24/02/2017 a 16/01/2020, abrangendo 723 dias úteis. Com o auxílio da ferramenta "Calculadora do Cidadão", módulo "Correção de Valores", disponível na página do $B C B^{2}$ (Banco Central do Brasil), podemos obter a taxa DI acumulada nesse período

\footnotetext{
${ }^{2}$ https://www.bcb.gov.br/.
} 
em 21,839389\%, bem como seu valor corrigido pelo percentual $q$, correspondente a 25,502889\%. Pela equação (4):

$$
\hat{p}_{2}=\frac{(1-0,15) \cdot 0,25502889}{0,21839389}=0,992585262 \quad \text { ou } \quad 99,2585262 \%
$$

Assim, estimamos que o investimento em questão rendeu, após o desconto do imposto de renda, cerca de $99,26 \%$ do DI.

No Exemplo 2 utilizamos um período do passado e, na prática, quando o objetivo for a tomada de decisão entre dois investimentos pós-fixados com tributação distinta, a taxa $i_{j}$ será desconhecida. Assim, para utilizá-la é necessário especificar uma taxa média diária (aquela que se espera para o período futuro), de modo que a equação (4) se torna:

$$
\hat{p}_{2}=\frac{\left(1-i_{r}\right)\left[(1+q i)^{n}-1\right]}{(1+i)^{n}-1} .
$$

Exemplo 3. Considere dois produtos de Renda Fixa pós-fixados, ambos com o mesmo prazo de 723 dias (úteis) de aplicação: o primeiro tributado e indexado em $115 \%$ do DI, já o segundo sem tributação. Se esperarmos que a taxa de juros média no período permaneça em torno de 4,5\% a.a., então a fórmula (5) pode ser utilizada para obtermos uma estimativa do percentual $p$, de acordo com o qual o segundo investimento deve remunerar para que seja preferível ao primeiro:

$$
\hat{p}_{2}=\frac{(1-0,15)\left\{\left[1+1,15\left((1+0,045)^{1 / 252}-1\right)\right]^{723}-1\right\}}{(1+0,045)^{723 / 252}-1}=0,98699991
$$

ou 98,699991\%. Diante dessa estimativa, um produto pós-fixado isento de imposto que pague algo superior a 98,70\% do DI deverá ser preferível, em termos de rendimento, ao produto que paga $115 \%$.

Os Exemplos 2 e 3 evidenciam duas dificuldades associadas às equações (4) e (5):

- Sua dependência da taxa $i$ futura: produtos pós-fixados não possuem taxa de juros conhecida, ocasião em que a utilização da fórmula (5) acaba sendo reduzida a algo semelhante ao apresentado no Exemplo 3, isto é, atribui-se um valor à taxa $i$, normalmente a taxa Selic corrente (não se pode, evidentemente, esperar que a taxa de 4,5\% a.a. se mantenha inalterada por 723 dias);

- Para que as equações (4) e (5) satisfaçam a Definição 2 de equivalência de percentuais, é necessário supor, de modo análogo ao ocorrido na seção 2.1, que o percentual $p$ incida diretamente sobre a taxa de juros acumulada no período e, como já mencionado na mesma seção, isso não ocorre na prática. 


\subsection{Relação Exata Para a Equivalência de Percentuais}

Os dois estimadores apresentados até o momento estão alicerçados numa hipótese que não é válida: a incidência do percentual destacado $p$ sobre a taxa acumulada $i_{a}$. Para estabelecermos a correta relação de equivalência entre $p$ e $q$, nos termos da Definição 2 e da equação (3), definida em [4], gostaríamos que os rendimentos líquidos associados aos investimentos $P$ e $Q$, no período $n$, fossem os mesmos, isto é, para um certo capital $C$ :

$$
C\left[\prod_{j=1}^{n}\left(1+p i_{j}\right)-1\right]=J_{P}=J_{Q}=C\left[\prod_{j=1}^{n}\left(1+q i_{j}\right)-1\right]\left(1-i_{r}\right)
$$

ou seja, a relação entre $p$ e $q$ deve ser dada por:

$$
\prod_{j=1}^{n}\left(1+p i_{j}\right)=\left(1-i_{r}\right) \prod_{j=1}^{n}\left(1+q i_{j}\right)+i_{r}
$$

A equação (6) define a relação exata entre $p$ e $q$, a partir de $i_{j}, 1 \leq j \leq n$ e $i_{r}$. No caso de $q$ ser conhecido, uma equação polinomial de grau $n$ na variável $p$ se torna definida. A seguir analisamos o Exemplo 2 à luz da equação (6).

Exemplo 4. No Exemplo 2 analisamos um produto de renda fixa pós-fixado em 115\% do DI durante o período de 24/02/2017 a 16/01/2020, compreendendo 723 dias úteis. Como se trata de um período passado, as taxas $i_{j}, 1 \leq j \leq 723$ são conhecidas e, por isso, a equação (6) pode ser utilizada, ou seja, o valor real de $p$ pode ser obtido. A referida equação foi resolvida numericamente pelo Método de Newton-Raphson, definido por [3]:

$$
p^{(k+1)}=p^{(k)}-\frac{f\left(p^{(k)}\right)}{f^{\prime}\left(p^{(k)}\right)}, \quad k \geq 1,
$$

em que

$$
f(p)=\prod_{j=1}^{n}\left(1+p i_{j}\right)-\left(1-i_{r}\right) \prod_{j=1}^{n}\left(1+q i_{j}\right)-i_{r}
$$

e $f^{\prime}(p)$ é a derivada de $f$ no ponto $p$. O método foi implementado ${ }^{3}$ na linguagem

\footnotetext{
${ }^{3}$ Adotamos a estimativa inicial de $p^{(1)}=\left(1-i_{r}\right) q$ e o critério de parada $\left|p^{(k)}-p^{(k-1)}\right| \leq 10^{-12}$. Com o estimador de erro a posteriori [7]:

$$
\epsilon\left(p^{(k)}\right)=\left|p^{(k)}-p^{*}\right| \leq \frac{M}{2 m}\left|p^{(k)}-p^{(k-1)}\right|^{2}, \quad m=\min _{x \in[a, b]}\left|f^{\prime}(x)\right|, \quad M=\max _{x \in[a, b]}\left|f^{\prime \prime}(x)\right|,
$$

estimamos que o erro a partir da solução verdadeira $p^{*}$, no intervalo $[a, b]=\left[\left(1-i_{r}\right) q, q\right]$, deve ser de no máximo $1,15232244969 \times 10^{-28}$.
} 
python e o valor obtido para p foi de 0,993266223147 ou $99,3266223147 \%$.

Comparando os resultados dos Exemplos 2 e 4, vemos que o estimador $\hat{p}_{2}$ produz uma boa aproximação para p, diferindo em cerca de 0,068557767\%.

É importante notar, no entanto, que de maneira análoga ao ocorrido na seção 2.2, a equação (6) carrega consigo a dificuldade de sua dependência da taxa futura. Uma simplificação que favorece seu uso consiste em admitir uma taxa DI diária constante $i=i_{j}$, para todo $j=1,2, \cdots, n$. Neste caso, a equação (6) se torna

$$
(1+p i)^{n}=\left(1-i_{r}\right)(1+q i)^{n}+i_{r},
$$

e com isso,

$$
\hat{p}_{3}=\frac{\sqrt[n]{(1+q i)^{n}\left(1-i_{r}\right)+i_{r}}-1}{i}
$$

onde, embora (8) seja solução exata de (7), utilizamos o símbolo $\hat{p}_{3}$ para denotar que se trata de um estimador para $p$, uma vez que a hipótese de taxa diária constante não condiz com a realidade.

Exemplo 5. Podemos analisar o Exemplo 3 à luz do estimador (8):

$$
\hat{p}_{3}=\frac{\sqrt[723]{(1-0,15)\left[1+1,15\left((1+0,045)^{1 / 252}-1\right)\right]^{723}+0,15}-1}{(1+0,045)^{1 / 252}-1}=0,987776805
$$

ou $98,7776805 \%$.

Com o Exemplo 5 verificamos que os estimadores (5) e (8), além das limitações em comum, produzem resultados muito próximos. Na seção a seguir exploraremos as relações entre os três estimadores apresentados.

\section{Relação Entre os Estimadores}

Notamos que se $n=1$, então as equações (2), (5) e (8) se tornam idênticas e, por essa razão, consideraremos nos resultados a seguir $2 \leq n \in \mathbb{N}$. Os teoremas a seguir mostram que as relações entre $\hat{p}_{1}, \hat{p}_{2}$ e $\hat{p}_{3}$ dependem da magnitude do percentual $q$.

Teorema 1. Se a taxa $i$ assume valores dentro do intervalo [0,1], então:

i) $S e q=1$, então $\hat{p}_{2}=\hat{p}_{1}$;

ii) $S e 0<q<1$ e $n \geq 2$, então $\hat{p}_{2}<\hat{p}_{1}$;

iii) Se $q>1$ e $n \geq 2$, então $\hat{p}_{2}>\hat{p}_{1}$. 
Demonstração. A partir da equação (5), a implicação i) é obvia. Para os demais casos começamos por utilizar o Teorema Binomial a fim de obtermos a seguinte equação:

$$
\left[(1+q i)^{n}-1\right]-q\left[(1+i)^{n}-1\right]=\sum_{k=2}^{n}\left(\begin{array}{l}
n \\
k
\end{array}\right) i^{k}\left(q^{k}-q\right), \quad n \geq 2 .
$$

Agora, comparando as implicações

$$
\left\{\begin{array}{cll}
0<q<1 & \Rightarrow & q^{k}<q, \quad k \geq 2, \\
q>1 & \Rightarrow \quad q^{k}>q, \quad k \geq 2,
\end{array}\right.
$$

com a equação (9), dado que $i>0$, concluímos que

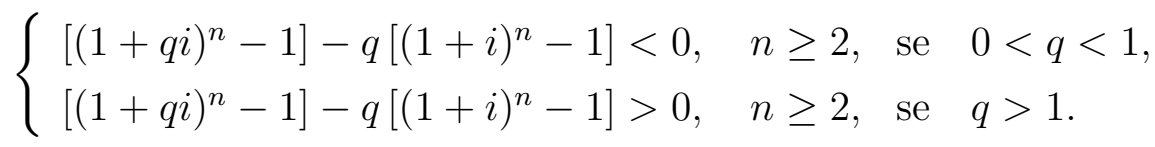

Comparando (11) e (5), concluímos que

$$
\left\{\begin{array}{l}
\hat{p}_{2}<\hat{p}_{1}, \quad \text { se } \quad 0<q<1, n \geq 2, \\
\hat{p}_{2}>\hat{p}_{1}, \quad \text { se } \quad q>1, n \geq 2 .
\end{array}\right.
$$

Teorema 2. O percentual $\hat{p}_{1}$ limita inferiormente $\hat{p}_{3}$, isto é, $\hat{p}_{1}<\hat{p}_{3}$ para $n \geq 2$.

Demonstração. Mostraremos primeiro que:

$$
\left(1-i_{r}\right)(1+q i)^{n}+i_{r}>\left[1+\left(1-i_{r}\right) q i\right]^{n}, \quad n \geq 2 .
$$

Com efeito, a equação (13) é verdadeira para $n=2$, pois

$$
\begin{aligned}
\left(1-i_{r}\right)(1+q i)^{2}+i_{r} & =(1+q i)^{2}-2(1+q i) i_{r} q i+i_{r}(q i)^{2}, \\
& >(1+q i)^{2}-2(1+q i) i_{r} q i+\left(i_{r} q i\right)^{2}, \\
& =\left[1+\left(1-i_{r}\right) q i\right]^{2},
\end{aligned}
$$

onde na primeira passagem utilizamos o fato de $0<i_{r}<1$ implicar que $i_{r}>i_{r}^{2}$. Supondo que (13) seja válida para $n=k$. Então:

$$
\left(1-i_{r}\right)(1+q i)^{k+1}+i_{r}=\left(1-i_{r}\right)(1+q i)^{k}+i_{r}+(1+q i)^{k}\left(1-i_{r}\right) q i .
$$


Pela hipótese de indução, temos que a equação (15) implica a inequação

$$
\left(1-i_{r}\right)(1+q i)^{k+1}+i_{r}>\left[1+\left(1-i_{r}\right) q i\right]^{k}+(1+q i)^{k}\left(1-i_{r}\right) q i .
$$

Uma vez que $0<i_{r}<1$ e por isso, $1>1-i_{r}$, temos então que

$$
(1+q i)^{n}>\left[1+\left(1-i_{r}\right) q i\right]^{n}, \quad 1 \leq n \in \mathbb{N} .
$$

Assim, combinando as inequações (16) e (17), obtemos

$$
\begin{aligned}
\left(1-i_{r}\right)(1+q i)^{k+1}+i_{r} & >\left[1+\left(1-i_{r}\right) q i\right]^{k}+\left[1+\left(1-i_{r}\right) q i\right]^{k}\left(1-i_{r}\right) q i \\
& =\left[1+\left(1-i_{r}\right) q i\right]^{k+1}
\end{aligned}
$$

Dessa forma, (13) é verdadeira e combinando-a com a equação (7) chegamos à

$$
\left(1+\hat{p}_{3} i\right)^{n}>\left[1+\left(1-i_{r}\right) q i\right]^{n}, \quad n \geq 2,
$$

isto é,

$$
\hat{p}_{3}>\left(1-i_{r}\right) q, \quad n \geq 2 \quad \text { ou } \quad \hat{p}_{3}>\hat{p}_{1}, \quad n \geq 2 .
$$

É resultado imediato dos Teoremas 1 e 2 o seguinte

Corolário 1. Se $q<1$ e $n \geq 2$, então $\hat{p}_{2}<\hat{p}_{3}$.

Demonstração. De fato, os Teoremas 1 e 2 nos dizem, respectivamente, que $\hat{p}_{2}<\hat{p}_{1}$ e $\hat{p}_{1}<\hat{p}_{3}$, quando $q \leq 1$ e $n \geq 2$. Assim, $\hat{p}_{2}<\hat{p}_{3}$ sempre que $q<1$ e $n \geq 2$.

O teorema a seguir estabelece outros critérios que relacionam $\hat{p}_{2}$ e $\hat{p}_{3}$.

Teorema 3. Se $\hat{p}_{3}>1$ (ou $\hat{p}_{2}>1$ ), então $\hat{p}_{2}>\hat{p}_{3}$. Se, ao contrário, $\hat{p}_{3}<1$ (ou $\left.\hat{p}_{2}<1\right)$, então $\hat{p}_{2}<\hat{p}_{3}$.

Demonstração. Das equações (5) e (7) obtemos a relação explícita:

$$
\hat{p}_{2}=\frac{\left(1+\hat{p}_{3} i\right)^{n}-1}{(1+i)^{n}-1}, \quad \text { ou ainda, } \quad \hat{p}_{2}\left[(1+i)^{n}-1\right]=\left(1+\hat{p}_{3} i\right)^{n}-1
$$

Comparando as relações anteriores com as desigualdades em (11), concluímos que:

$$
\left\{\begin{array} { l } 
{ \hat { p } _ { 2 } > \hat { p } _ { 3 } , \quad \text { se } \hat { p } _ { 3 } > 1 , n \geq 2 , } \\
{ \hat { p } _ { 2 } < \hat { p } _ { 3 } , \quad \text { se } \hat { p } _ { 3 } < 1 , n \geq 2 , }
\end{array} \quad \text { e } \quad \left\{\begin{array}{l}
\hat{p}_{2}>\hat{p}_{3}, \quad \text { se } \hat{p}_{2}>1, n \geq 2 \\
\hat{p}_{2}<\hat{p}_{3}, \quad \text { se } \hat{p}_{2}<1, n \geq 2
\end{array}\right.\right.
$$


respectivamente.

A seguir estabelecemos o limitante superior de $\hat{p}_{3}$.

Teorema 4. A sequência $\left(\left(\hat{p}_{3}\right)_{n}\right)_{n \in \mathbb{N}}$, com n-ésimo termo $\left(\hat{p}_{3}\right)_{n}$ dado pela equação (8), converge para $q$.

Demonstração. Com efeito:

$$
\begin{aligned}
\lim _{n \rightarrow \infty}\left(\hat{p}_{3}\right)_{n} & =\lim _{n \rightarrow \infty} \frac{\sqrt[n]{(1+q i)^{n}\left(1-i_{r}\right)+i_{r}}-1}{i} \\
& =\lim _{n \rightarrow \infty}\left\{\frac{1+q i}{i} \sqrt[n]{1-i_{r}+\frac{i_{r}}{(1+q i)^{n}}}-\frac{1}{i}\right\}
\end{aligned}
$$

uma vez que, quando $n$ tende ao infinito, $\sqrt[n]{1-i_{r}+i_{r}(1+q i)^{-n}}$ se aproxima de 1 , dado que $1-i_{r}>0$.

O resultado anterior estabelece que se os percentuais $q, i$ e $i_{r}$ forem constantes, então para períodos $n$ muito grandes (tendendo ao infinito), o percentual $\hat{p}_{3}$ se torna muito próximo de $q$. Como $\left(\left(\hat{p}_{3}\right)_{n}\right)_{n \in \mathbb{N}}$ é monótona crescente (o leitor pode verificar isso), segue-se que $\hat{p}_{3}$ é limitado superiormente por $q$. Outra maneira de verificar isso é notar que:

$$
1+q i>1 \Rightarrow(1+q i)^{n}>1, n \in \mathbb{N} \quad \Rightarrow \quad-i_{r}(1+q i)^{n}<-i_{r}, n \in \mathbb{N},
$$

e com isso

$$
(1+q i)^{n}-i_{r}(1+q i)^{n}+i_{r}<(1+q i)^{n}-i_{r}+i_{r}=(1+q i)^{n}, n \in \mathbb{N} .
$$

Dessa forma, obtemos que

$$
\hat{p}_{3}<\frac{\sqrt[n]{(1+q i)^{n}}-1}{i}=q
$$

Em termos práticos, é evidente que o percentual $p$, associado a investimentos isentos, não pode superar, em termos de equivalências, o percentual q, que remunera investimentos tributados.

Diante destes resultados, obtemos que

$$
\left(1-i_{r}\right) q \leq \hat{p}_{3}<q
$$


O estimador $\hat{p}_{2}$ se torna limitado (por $q$ ) apenas no caso em que $q \leq 1$ (basta combinar a equação (27) e o Corolário 1). Para $q>1$, por outro lado, a sequência $\left(\left(\hat{p}_{2}\right)_{n}\right)_{n \in \mathbb{N}}$, dada pela equação (5), é monótona crescente (o leitor pode provar isso) e ilimitada. De fato, tomando o limite e notando que $(1+i) /(1+q i)<1$ :

$\lim _{n \rightarrow \infty}\left(\hat{p}_{2}\right)_{n}=\left(1-i_{r}\right) \lim _{n \rightarrow \infty} \frac{(1+q i)^{n}-1}{(1+i)^{n}-1}=\left(1-i_{r}\right) \lim _{n \rightarrow \infty} \frac{1-\frac{1}{(1+q i)^{n}}}{\left(\frac{1+i}{1+q i}\right)^{n}-\frac{1}{(1+q i)^{n}}}=\infty$.

Desta forma, podemos resumir os resultados deduzidos nesta seção da seguinte maneira:

$$
\begin{aligned}
\hat{p}_{2}<\hat{p}_{1}<\hat{p}_{3}<q, & \text { se } q<1, n \geq 2, \\
\hat{p}_{1}<\hat{p}_{2}<\hat{p}_{3}<q, & \text { se } q>1, \text { enquanto } \hat{p}_{2}<1, \\
\hat{p}_{1}<\hat{p}_{3}<\hat{p}_{2}, & \text { se } q>1, \text { enquanto } \hat{p}_{2}>1 .
\end{aligned}
$$

Na seção seguinte apresentamos simulações computacionais em linguagem python construídas a partir da base de dados disponível em [4].

\section{Experimentos Computacionais}

As simulações apresentadas nesta seção, desenvolvidas a partir da série histórica da Taxa DI, formada pelo período compreendido entre as datas ${ }^{4}$ de 02/01/1998 e $14 / 01 / 2020$, tem a finalidade de investigar o comportamento dos estimadores $\hat{p}_{1}, \hat{p}_{2}$ e $\hat{p}_{3}$ em detrimento do verdadeiro valor do percentual $p$.

Realizamos três experimentos computacionais simulando um investimento pósfixado com remuneração de $q=115 \%$ da Taxa DI, com períodos de aplicação de 180, 360 e 720 dias, cujos resultados estão expressos pelas Figuras 1, 2 e 3 e Tabelas 2, 3 e 4, respectivamente. Como visto na seção 3 , a utilização de $\hat{p}_{2}$ e $\hat{p}_{3}$ depende da definição de uma taxa média esperada para o período. Consideramos, portanto, três formas de se obter essa taxa:

i) Tomando a taxa média $i_{m}$ do período imediatamente anterior, isto é, dos últimos

\footnotetext{
${ }^{4} \mathrm{O}$ banco de dados é atualizado diariamente, razão pela qual sua extensão deve ser maior, a depender da data da pesquisa.
} 
180, 360 ou 720 dias, conforme o período da simulação:

$$
i_{m}=\sqrt[t]{\prod_{i=1}^{t}\left(1+i_{j}\right)}-1, \quad t=180,360 \text { ou } 720
$$

abordagem essa que gerou os gráficos das Figuras 1a, 2a e 3a;

ii) Utilizando a Taxa DI do dia anterior ao início da aplicação, simulando o uso da taxa corrente no momento da aplicação, conforme resultados dispostos nas Figuras 1b, 2b e 3b;

iii) Utilizando médias móveis para estimar a taxa pertinente ao período de aplicação, estratégia esta que forneceu os gráficos das Figuras 1c, 2c e 3c.

Figura 1: Percentual $p$ e suas estimativas $\hat{p}_{1}, \hat{p}_{2}$ e $\hat{p}_{3}$ obtidas nas simulações de investimentos realizados entre as datas de 23/09/1998 e 02/05/2019, pós-fixados em $q=115 \%$ do DI por prazos de 180 dias.

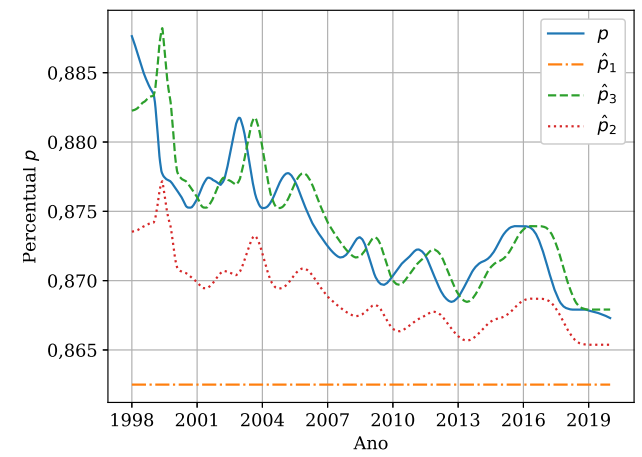

(a) Estimativas pela taxa média do período passado.

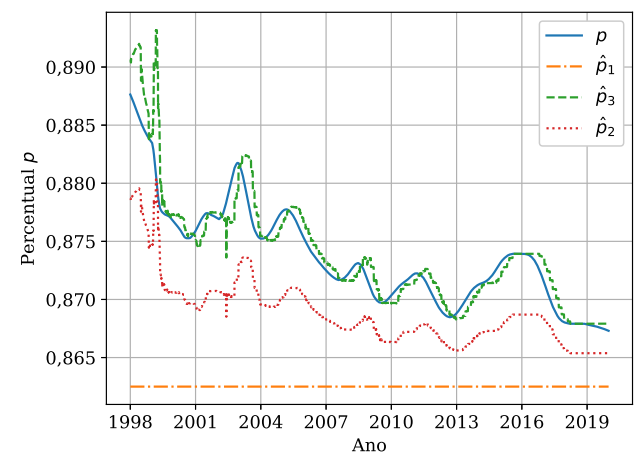

(b) Estimativas pela taxa do dia anterior.

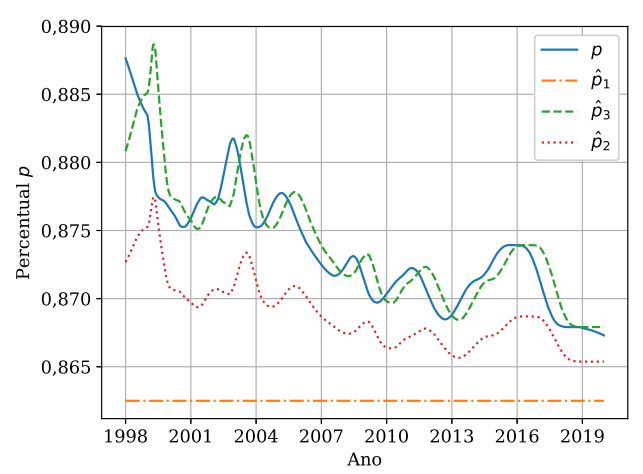

(c) Estimativas pela média móvel do período passado.

Fonte: Autoral. 
Tabela 2: Erro médio, desvio padrão e erro máximo cometido pelos estimadores $\hat{p}_{1}$, $\hat{p}_{2}$ e $\hat{p}_{3}$ ao se aproximar o percentual $p$, nas simulações de investimentos realizados entre as datas de 23/09/1998 e 02/05/2019, pós-fixados em $q=115 \%$ do DI por prazos de 180 dias.

\begin{tabular}{|c|c|c|c|c|c|c|c|}
\hline \multirow{3}{*}{ Estatística } & \multirow[b]{3}{*}{$\hat{p}_{1}$} & \multicolumn{6}{|c|}{ Estratégia para a estimativa da taxa $i$} \\
\hline & & \multicolumn{2}{|c|}{ Taxa Média } & \multicolumn{2}{|c|}{ Taxa do último dia } & \multicolumn{2}{|c|}{ Média Móvel } \\
\hline & & $\hat{p}_{2}$ & $\hat{p}_{3}$ & $\hat{p}_{2}$ & $\hat{p}_{3}$ & $\hat{p}_{2}$ & $\hat{p}_{3}$ \\
\hline Erro Médio & $1,1 \times 10^{-2}$ & $4,7 \times 10^{-3}$ & $1,8 \times 10^{-3}$ & $4,8 \times 10^{-3}$ & $1,1 \times 10^{-3}$ & $4,7 \times 10^{-3}$ & $1,6 \times 10^{-3}$ \\
\hline Desv. Padrão & $3,8 \times 10^{-3}$ & $2,2 \times 10^{-3}$ & $1,6 \times 10^{-3}$ & $1,8 \times 10^{-3}$ & $1,3 \times 10^{-3}$ & $2,1 \times 10^{-3}$ & $1,5 \times 10^{-3}$ \\
\hline Erro Máximo & $2,5 \times 10^{-2}$ & $1,4 \times 10^{-2}$ & $1,1 \times 10^{-2}$ & $1,1 \times 10^{-2}$ & $1,3 \times 10^{-2}$ & $1,5 \times 10^{-2}$ & $1,1 \times 10^{-2}$ \\
\hline
\end{tabular}

Fonte: Autoral.

Figura 2: Percentual $p$ e suas estimativas $\hat{p}_{1}, \hat{p}_{2}$ e $\hat{p}_{3}$ obtidas nas simulações de investimentos realizados entre as datas de 16/06/1999 e 09/08/2018, pós-fixados em $q=115 \%$ do DI por prazos de 360 dias.

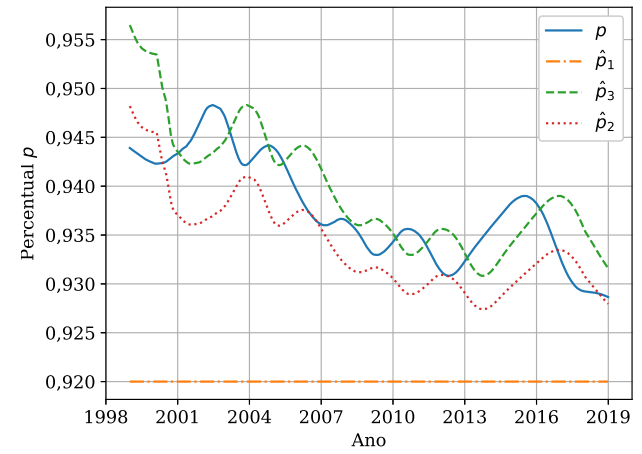

(a) Estimativas pela média do período passado.

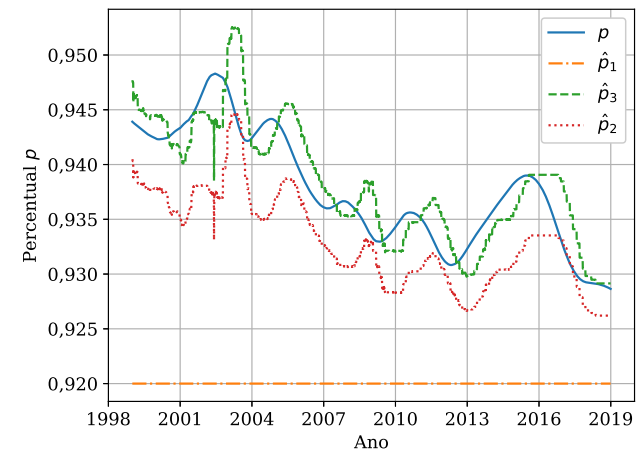

(b) Estimativas pela taxa do dia anterior.

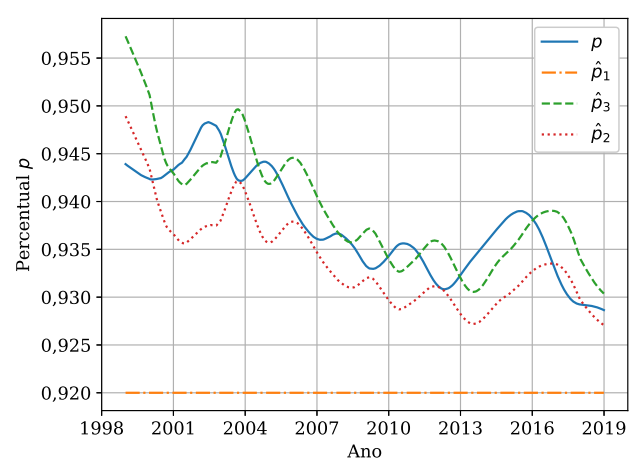

(c) Estimativas pela média móvel do período passado.

Fonte: Autoral. 
Tabela 3: Erro médio, desvio padrão e erro máximo cometido pelos estimadores $\hat{p}_{1}$, $\hat{p}_{2}$ e $\hat{p}_{3}$ ao se aproximar o percentual $p$, nas simulações de investimentos realizados entre as datas de 16/06/1999 e 09/08/2018, pós-fixados em $q=115 \%$ do DI por prazos de 360 dias.

\begin{tabular}{|c|c|c|c|c|c|c|c|}
\hline \multirow{3}{*}{ Estatística } & \multirow[b]{3}{*}{$\hat{p}_{1}$} & \multicolumn{6}{|c|}{ Estratégia para a estimativa da taxa $i$} \\
\hline & & \multicolumn{2}{|c|}{ Taxa Média } & \multicolumn{2}{|c|}{ Taxa do último dia } & \multicolumn{2}{|c|}{ Média Móvel } \\
\hline & & $\hat{p}_{2}$ & $\hat{p}_{3}$ & $\hat{p}_{2}$ & $\hat{p}_{3}$ & $\hat{p}_{2}$ & $\hat{p}_{3}$ \\
\hline Erro Médio & $1,8 \times 10^{-2}$ & $4,4 \times 10^{-3}$ & $3,7 \times 10^{-3}$ & $4,7 \times 10^{-3}$ & $2,4 \times 10^{-3}$ & $4,5 \times 10^{-3}$ & $3,4 \times 10^{-3}$ \\
\hline Desv. Padrão & $5,2 \times 10^{-3}$ & $3,1 \times 10^{-3}$ & $2,6 \times 10^{-3}$ & $2,6 \times 10^{-3}$ & $1,7 \times 10^{-3}$ & $3,0 \times 10^{-3}$ & $2,4 \times 10^{-3}$ \\
\hline Erro Máximo & $2,8 \times 10^{-2}$ & $1,1 \times 10^{-2}$ & $1,3 \times 10^{-2}$ & $1,5 \times 10^{-2}$ & $9,8 \times 10^{-3}$ & $1,1 \times 10^{-2}$ & $1,3 \times 10^{-2}$ \\
\hline
\end{tabular}

Fonte: Autoral.

Figura 3: Percentual $p$ e suas estimativas $\hat{p}_{1}, \hat{p}_{2}$ e $\hat{p}_{3}$ obtidas nas simulações de investimentos realizados entre as datas de 20/11/2000 e 02/03/2017, pós-fixados em $q=115 \%$ do DI por prazos de 720 dias.

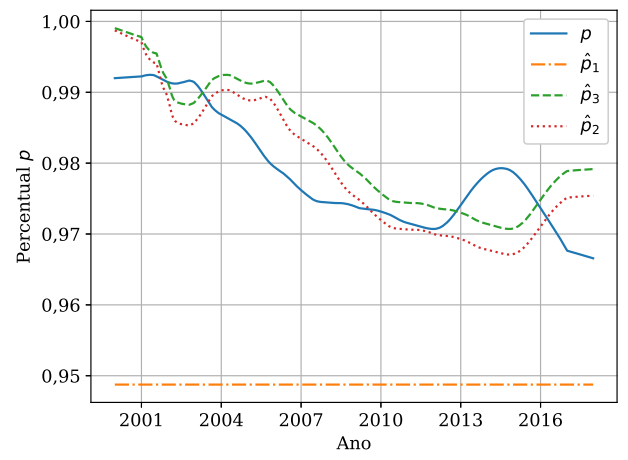

(a) Estimativas pela taxa média do período passado.



(b) Estimativas pela taxa do dia anterior.

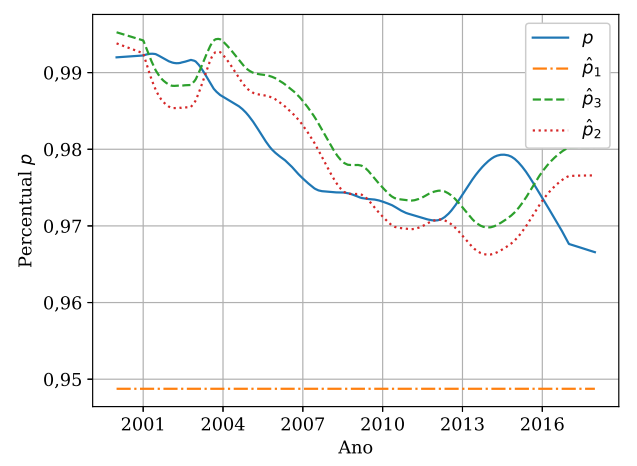

(c) Estimativas pela média móvel do período passado.

Fonte: Autoral.

As simulações foram arquitetadas da seguinte forma: para cada dia $d$ da série histórica $S_{n \times 1}$, foi suposto se ter investido sobre uma remuneração de $q=115 \%$ do DI, por um período $t=180,360$ ou 720 dias, conforme cada caso (Figuras 1, 2 e 3, 
respectivamente).

Tabela 4: Erro médio, desvio padrão e erro máximo cometido pelos estimadores $\hat{p}_{1}$, $\hat{p}_{2}$ e $\hat{p}_{3}$ ao se aproximar o percentual $p$, nas simulações de investimentos realizados entre as datas de 20/11/2000 e 02/03/2017, pós-fixados em $q=115 \%$ do DI por prazos de 720 dias.

\begin{tabular}{|c|c|c|c|c|c|c|c|}
\hline \multirow{3}{*}{ Estatística } & \multirow[b]{3}{*}{$\hat{p}_{1}$} & \multicolumn{6}{|c|}{ Estratégia para a estimativa da taxa $i$} \\
\hline & & \multicolumn{2}{|c|}{ Taxa Média } & \multicolumn{2}{|c|}{ Taxa do último dia } & \multicolumn{2}{|c|}{ Média Móvel } \\
\hline & & $\hat{p}_{2}$ & $\hat{p}_{3}$ & $\hat{p}_{2}$ & $\hat{p}_{3}$ & $\hat{p}_{2}$ & $\hat{p}_{3}$ \\
\hline Erro Médio & $3,0 \times 10^{-2}$ & $4,7 \times 10^{-3}$ & $5,4 \times 10^{-3}$ & $5,2 \times 10^{-3}$ & $5,0 \times 10^{-3}$ & $4,6 \times 10^{-3}$ & $5,2 \times 10^{-3}$ \\
\hline Desv. Padrão & $7,2 \times 10^{-3}$ & $3,4 \times 10^{-3}$ & $3,2 \times 10^{-3}$ & $3,6 \times 10^{-3}$ & $3,4 \times 10^{-3}$ & $3,4 \times 10^{-3}$ & $3,1 \times 10^{-3}$ \\
\hline Erro Máximo & $4,4 \times 10^{-2}$ & $1,2 \times 10^{-2}$ & $1,3 \times 10^{-2}$ & $1,4 \times 10^{-2}$ & $1,3 \times 10^{-2}$ & $1,3 \times 10^{-2}$ & $1,4 \times 10^{-2}$ \\
\hline
\end{tabular}

Fonte: Autoral.

Figura 4: Percentual $p$ e suas estimativas $\hat{p}_{1}, \hat{p}_{2}$ e $\hat{p}_{3}$ obtidas nas simulações de investimentos pós-fixados em $q=98 \%$ do DI, com prazos de 90 (a) e 900 (b) dias e taxa $i_{m}$ estimada pela média do período anterior.

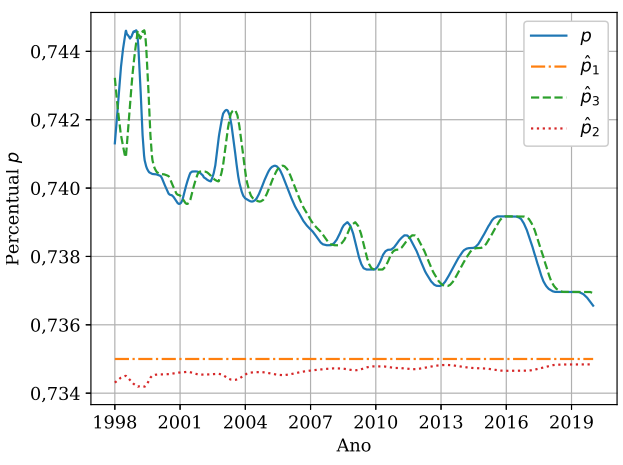

(a) $t=90$ dias.

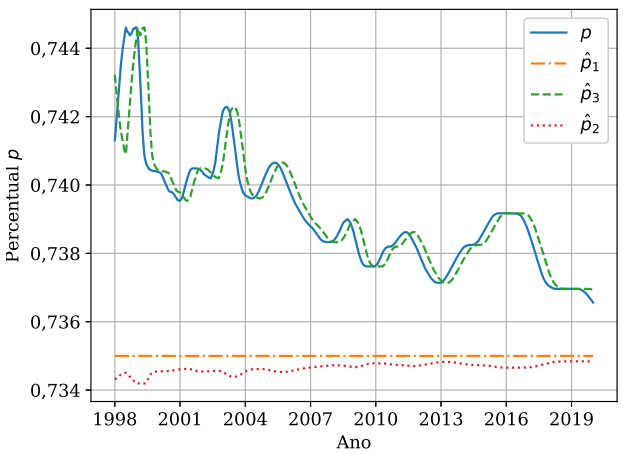

(c) Fig. 4a com $\hat{p}_{2}$ e $\hat{p}_{3}$ trasladados $t=90$ dias.

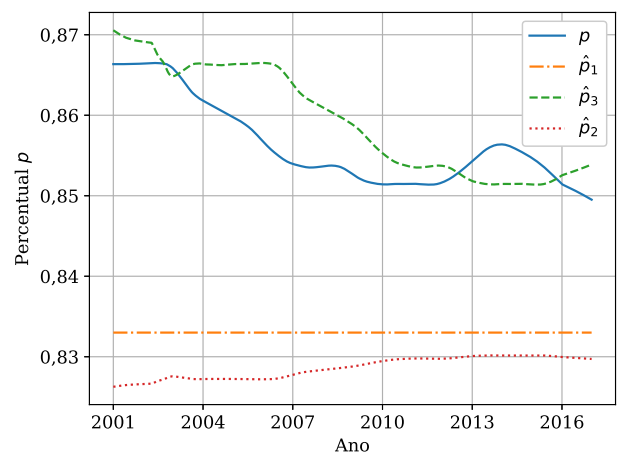

(b) $t=900$ dias.

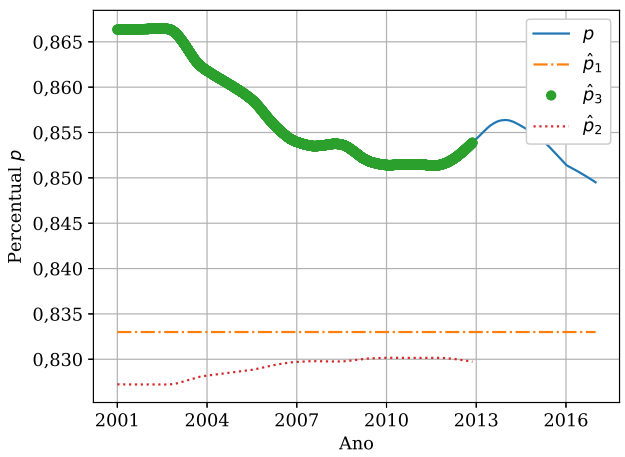

(d) Fig. $4 \mathrm{~b}$ com $\hat{p}_{2}$ e $\hat{p}_{3}$ trasladados $t=$ 900 dias.

Fonte: Autoral.

O percentual $p$ pôde ser estimado, pelo Método de Newton-Raphson ${ }^{5}$ analoga${ }^{5} \mathrm{O}$ critério de parada estabelecido neste caso foi $\left|p^{(k)}-p^{(k-1)}\right| \leq 10^{-6}$. 
mente ao exposto no Exemplo 4, porque as taxas dos dias seguintes eram conhecidas.

Obtido o percentual do dia $d$, repetia-se o cálculo para o próximo dia $d+1$, e assim sucessivamente até chegar ao limite que seria o dia $n-t$. A data na qual se iniciou a simulação foi $d=t+1$, assim definida pelo fato de que as estratégias para se obter a taxa $i$ envolvia ou uso da taxa média do período $t$ anterior ou o uso de médias móveis.

Os três períodos de aplicação simulados foram escolhidos com base na divisão que é própria da Tabela 1 de tributação de imposto sobre rendimentos de capital. Pelos gráficos observamos que as três estratégias utilizadas no cálculo de $i$ produzem estimativas com comportamento muito próximo. Além disso, a precisão dessas estimativas diminui conforme o prazo de aplicação o que é, no entanto, natural em qualquer processo de previsão.

Os gráficos constantes nas Figuras 1, 2 e 3 evidenciam que:

- O percentual $p$ não é estático e varia ao longo do tempo, conforme a oscilação da taxa de juros;

- A curva obtida pelo estimador $\hat{p}_{3}$, de modo geral, se aproxima da curva dada por $p$, a menos de um atraso no tempo correspondente ao período $t$. Isto ocorre em virtude de se estar utilizando os dados do período $t$ passado para se estimar o percentual devido ao período $t$ futuro (veja Figuras 4c e 4d);

- Quanto maior o prazo $t$ de aplicação mais suave se tornam as curvas $p$ e $\hat{p}_{3}$ (uma vez que esta última repete "com atraso $t$ " o padrão da primeira). Enfatizamos esse detalhe com a Figura 4, onde apresentamos dois gráficos com períodos de 90 e 900 dias (Figuras 4a e 4b, respectivamente), tomando $q=98 \%$ do DI.

As Tabelas 2, 3 e 4 mostram as estatísticas de erro médio, desvio padrão do erro e erro máximo para cada estimador e estratégia. As estatísticas tabeladas quantificam os dados qualitativos notados pelos gráficos e indicam que a dispersão em relação à média, acessada pelo desvio padrão, se torna maior (mesmo que levemente) a medida em que se aumenta o tempo de aplicação $t$.

\section{Conclusão}

Este trabalho buscou evidenciar as hipóteses que norteiam o desenvolvimento e a construção de algumas metodologias utilizadas para se determinar a relação de equivalência entre percentuais destacados para a remuneração de títulos pós-fixados. 
Utilizamos o termo "estimador" porque na prática nenhum deles fornece uma relação exata entre os percentuais (mesmo que a relação dada pela equação (6) seja exata, sua utilização na forma da equação (8) se torna dependente da taxa de juros que não é conhecida). Os erros médios registrados nas Tabelas 1, 2, 3 e 4 indicam que os três estimadores fornecem boas aproximações para o percentual $p$, porém, cada um deles possui sua vantagem e desvantagem:

- O estimador $\hat{p}_{1}$, dado pela equação (2), é caracterizado pela simplicidade e pelo fato de não se utilizar da taxa de juros. Esta última característica, no entanto, faz com que seu valor se torne constante, causando a impressão errada de que não há oscilações ao longo do tempo (conforme gráficos constantes nas Figuras 1, 2, 3 e 4);

- O estimador $\hat{p}_{2}$, obtido pela equação (5), considera a taxa de juros e por isso captura oscilações ao longo do tempo. Por outro lado, sua utilização é comprometida por necessitar da taxa de juros e também pelo fato de que sua dedução, assim como $\hat{p}_{1}$, se utiliza de uma hipótese inválida. Além disso, ao contrário de $\hat{p}_{1}$ e $\hat{p}_{3}$, esse estimador pode se tornar ilimitado quando $n$ cresce indefinidamente;

- O terceiro estimador $\hat{p}_{3}$, cuja fórmula é (8), é coerentemente deduzido por condições válidas, porém, sua utilização também depende da taxa de juros não conhecida.

Estes são os dois fatos que precisam estar claros quando se utiliza qualquer uma das três fórmulas de equivalência citadas neste texto: i) as conversões percentuais são aproximações e ii) seus valores verdadeiros flutuam ao longo do tempo, significando que um percentual $q$ convertido a $p$ em datas diferentes (digamos, hoje e daqui a um mês) pode possuir valores diferentes (esse é o significado das flutuações presentes nas curvas contínuas dispostas nos gráficos obtidos pelas simulações da seção 4).

\section{Agradecimentos}

Registramos nossos agradecimentos aos revisores que, com suas sugestões e apontamentos, no anonimato, contribuíram para a melhoria deste trabalho. 


\section{Referências}

[1] ASSAF NETO, A. Mercado Financeiro. 12a Ed. São Paulo: Editora Atlas, 2014.

[2] BANCO CENTRAL DO BRASIL (BC). Relatório de Economia Bancária. Disponível em: <https://www.bcb.gov.br/content/publicacoes/ relatorioeconomiabancaria/reb_2018.pdf $>$. Acesso em: 03 de fev. de 2020 .

[3] BURDEN, R. L.; FAIRES, J. D. Análise numérica. São Paulo: Cengage Learning, 2008.

[4] B3 (BRASIL BOLSA BALCÃO). Metodologia de Cálculo Acumulado. Disponível em: <http://www.b3.com.br/pt_br/ market-data-e-indices/indices/indices-de-segmentos-e-setoriais/ di/metodologia-de-calcudo-acumulado-de-di/>. Acesso em: 22 de jan. de 2020 .

[5] MARQUES, N. F. S. Estrutura e Funções do Sistema Financeiro no Brasil. Brasília: Thesaurus, 2003. p. 139 - 141.

[6] MINISTÉRIO DA ECONOMIA. Receita Federal. Disponível em: <http://receita.economia.gov.br/acesso-rapido/tributos/ irpf-imposto-de-renda-pessoa-fisica>. Acesso em: 21 de fev. de 2020.

[7] OSTROWSKI, A. M. Solution of equations and systems of equations. New York: Academic Press, 1966.

[8] PUCCINI, E. C. Matemática Financeira e Análise de Investimentos. Florianópolis: Departamento de Ciências da Administração / UFSC; [Brasília]: CAPES, UAB, 2011. 